\title{
Astronomical Research and Education in Tajikistan
}

\author{
Pulat B. Babadzhanov \\ Institute of Astrophysics, Dushanbe 734042, Tajikistan. e-mail: \\ pulat@astro.tajik.net
}

\begin{abstract}
Astronomical researches in Tajikistan are carried out by the Institute of Astrophysics, Tajik Academy of Sciences. The main scientific fields of investigation are physics and dynamics of asteroids, comets and meteors, variable stars and observations of artificial Earth satellites. The Institute has three observational stations: the Gissar observatory (at an altitude $730 \mathrm{~m}$ above sea-level) with photographic fireball and meteor patrols, a 70-cm reflector, a 40-cm Zeiss astrograph, a high-precision astronomical telescope $(\mathrm{D}=1 \mathrm{~m}, \mathrm{~F}=0.75 \mathrm{~m})$, etc.; the Sanglokh observatory (at an altitude $2300 \mathrm{~m}$ above sea-level) with a Ritchey-Chretien $1-\mathrm{m}$ telescope and $0.6-\mathrm{m}$ reflector by Carl Zeiss; the Pamir observatory (at an altitude $4350 \mathrm{~m}$ above sea-level) with a 70-cm reflector. In 1999 the Department of Astronomy was restored in the Tajik State National University and the first students were admitted to this university for the astronomical profession.
\end{abstract}

\section{Introduction}

From the most ancient times astronomy has occupied an important place in the culture of the peoples of Central Asia. Avesto contains the earliest items of information on the astronomical knowledge of the Central-Asian peoples. In the ninth to eleventh centuries in Central Asia, alongside mathematics, geography, medicine, philosophy and literature, astronomy flourished greatly. In the works of Aburayhan Beruni (973-1048), the greatest scientist of the tenth to eleventh centuries, detailed evidence of the general astronomical knowledge of the peoples of Central Asia of his period is given. The works of Aburayhan Beruni, Abu Ali ibn Sino (Avicenna, 980-1037), Abumahmud Khujandi (died about 1000), the designer of the sextant (the unique astronomical instrument of that epoch), Omar Khayyam (1048-1131), author of an original solar calendar (and famous poetry), Nasreddin Tusi (1201-1274), Mirzo Ulughbeg (1394-1449) and many talented scientists of the East, were an invaluable contribution in development of world astronomical science. The special development of astronomy in Central Asia was connected with creation of the famous astronomical observatory of Ulughbeg in Samarkand and with the formation of the Samarkand scientific school, where the greatest scientists, such as Qhozizodeh Rumi (1360 - 1437), Jamshed Koshi (died in 1430), Alauddin Ali Qhushchi (died in 1474), and others, worked actively. After Ulughbeg's death astronomical research in Central Asia gradually declined, but the ideas and traditions of the Samarkand scientists 
were continued by Ali Qhushchi and his pupils in Iran and Turkey. Modern astronomy in Tajikistan began in the 1930s, after the completion of the work of the Tajik-Pamir complex expedition (1928-1932) which recognized the favorable astroclimatic conditions and advantageous geographical location of Tajikistan. In 1932 the Tajikistan government accepted the decision to organize a Tajik astronomical observatory (TAO) and authorized its construction in the outskirts of Dushanbe. The principal directions of scientific research for the Observatory, namely, meteors, comets, and variable stars, were chosen taking into account the geographical location and climatic conditions of Tajikistan. The Observatory was to investigate the astroclimate of other parts of Tajikistan in order to choose the best site for a future high-mountain observatory. These directions, alongside others, remain as the principal ones to this day.

In 1948, TAO was renamed Stalinabad Astronomical Observatory (SAO). In 1958, on the base of the Observatory, the Institute of Astrophysics of the Tajik Academy of Sciences was created. It consisted of three departments:

Department of Meteor Astronomy,

Deparment of Comets,

Department of Variable Stars.

Afterwards the

Department of Theoretical Astrophysics (1962),

Laboratory of Experimental Astrophysics (1972), and

Department of Astrometry (1975) were created.

Some 30 years after the creation of the Institute, three of its modern observational bases were built:

1. In 1963-1971 the Gissar Astronomical Observatory (GissAO) was built at a distance of $14 \mathrm{~km}$ south-west of Dushanbe. Its domes house: a 70-cm reflector supplied with electron-optical, electrophotometric and polarimetric receiving apparatus, intended for observations of variable stars and comets; a 40-cm Zeiss astrograph for observations of asteroids, comets and variable stars; a 20-cm refractor and double astrograph. The observatory is also equipped with a high-precision astronomical camera $(D=1 \mathrm{~m}, \mathrm{~F}=0.75 \mathrm{~m})$ for photographic observation of artificial Earth satellites, comets and meteors. One of largest photographic meteor patrols is established in Gissar observatory. It consists of 16 wide-field cameras MK-75 $(\mathrm{F}=75 \mathrm{~cm} \mathrm{D} / \mathrm{F}=1: 3.5)$ and 24 cameras MK-25 $(\mathrm{F}=25 \mathrm{~cm}, \mathrm{D} / \mathrm{F}=1: 2.5)$. Observations of meteors are carried out also with TV and fireball cameras equipped with "fish-eye" lenses.

2. Sanglokh Observatory, the construction of which was completed in 1980, is located south-west of Dushanbe at a distance of about $90 \mathrm{~km}$. It was built at the top of Sanglokh Mountain, the astroclimatic conditions of which have been widely recognized (Table 1 ). A Ritchey-Chretien 1-m telescope equipped with a spectrograph, "UAGS", and photometer-polarimeter were set up at this Observatory. A $60-\mathrm{cm}$ Zeiss telescope is being assembled.

3. Pamir High-Mountain Observatory, the so-called "Solar ground-based astronomical complex Pamir" (situated at an altitude of $4350 \mathrm{~m}$ above sea level and enjoying 250 clear nights per year). It used to belong to the Main Astronomical Observatory (Pulkovo) of the Russian Academy of Sciences and was nationalized in 1991. It is located in the Murgab district (East Pamir) of the 


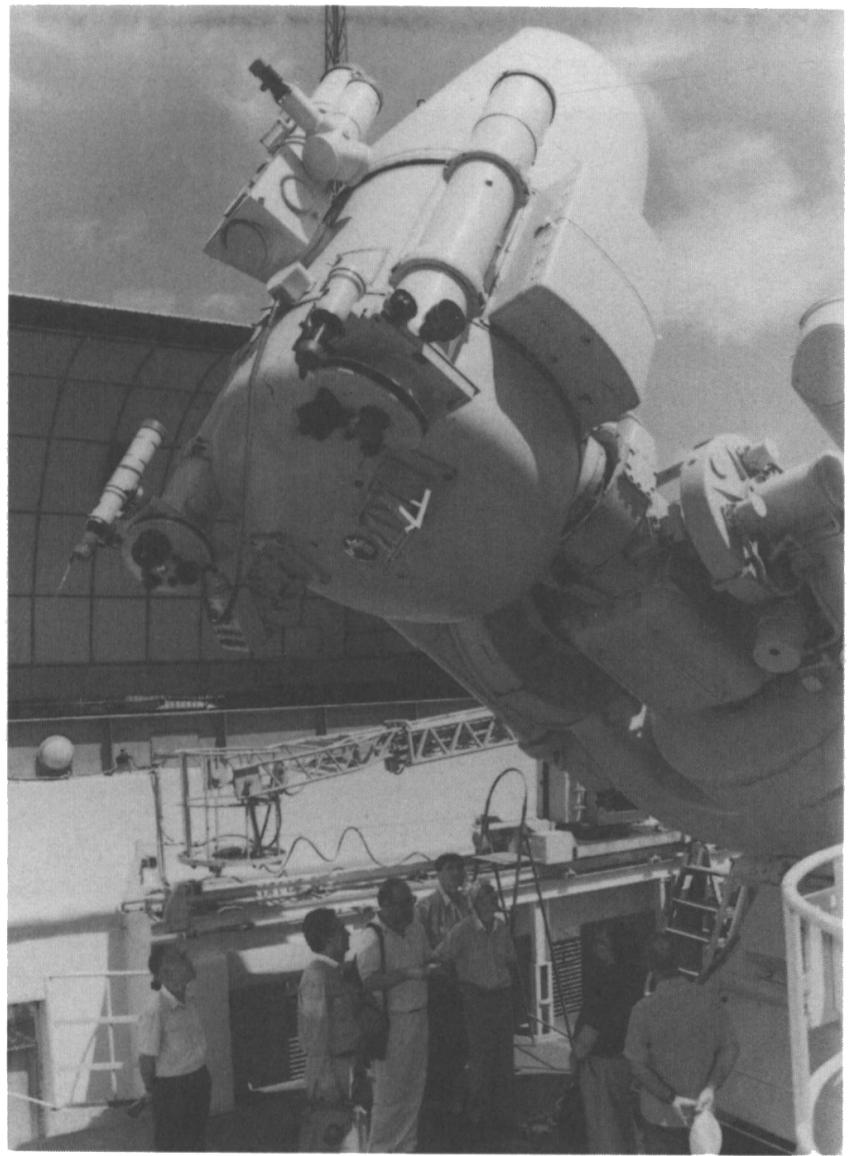

Figure 1. Gissar Observatory: The 1-m astronomical camera

Table 1. Astroclimatic Characteristics of the Astronomical Observatories of Tajikistan and some other Countries

\begin{tabular}{lrrrrrr}
\hline Observatory & Longitude & Latitude & Height $^{1}$ & $\mathrm{~N}^{2}$ & $\mathrm{~A}^{\prime \prime 3}$ & $\mathrm{Op}^{4}$ \\
\hline Sanglokh & 69.0 & 38.2 & 2300 & 1700 & 0.54 & 0.78 \\
Pamir & $74 .$. & $38 .$. & 4350 & 1820 & 0.54 & 0.86 \\
GissAO & 68.6 & 38.5 & 730 & 1620 & 2.10 & 0.72 \\
La Silla & -70.7 & -29.3 & 2347 & 2100 & 0.60 & 0.78 \\
Crimea & 34.0 & 44.6 & 600 & 1219 & 2.12 & 0.73 \\
Kitt Peak & -111.6 & 32.0 & 2120 & 2100 & 1.20 & 0.78 \\
Palomar & -116.9 & 33.4 & 1706 & 1706 & 2.00 & 0.80 \\
\hline
\end{tabular}

1 in metres above sea level; ${ }^{2}$ number of astronomicla hours per year; ${ }^{3}$ astroclimatic index (image quality in arcsec); ${ }^{4}$ optical transmission factor of the atmosphere. 


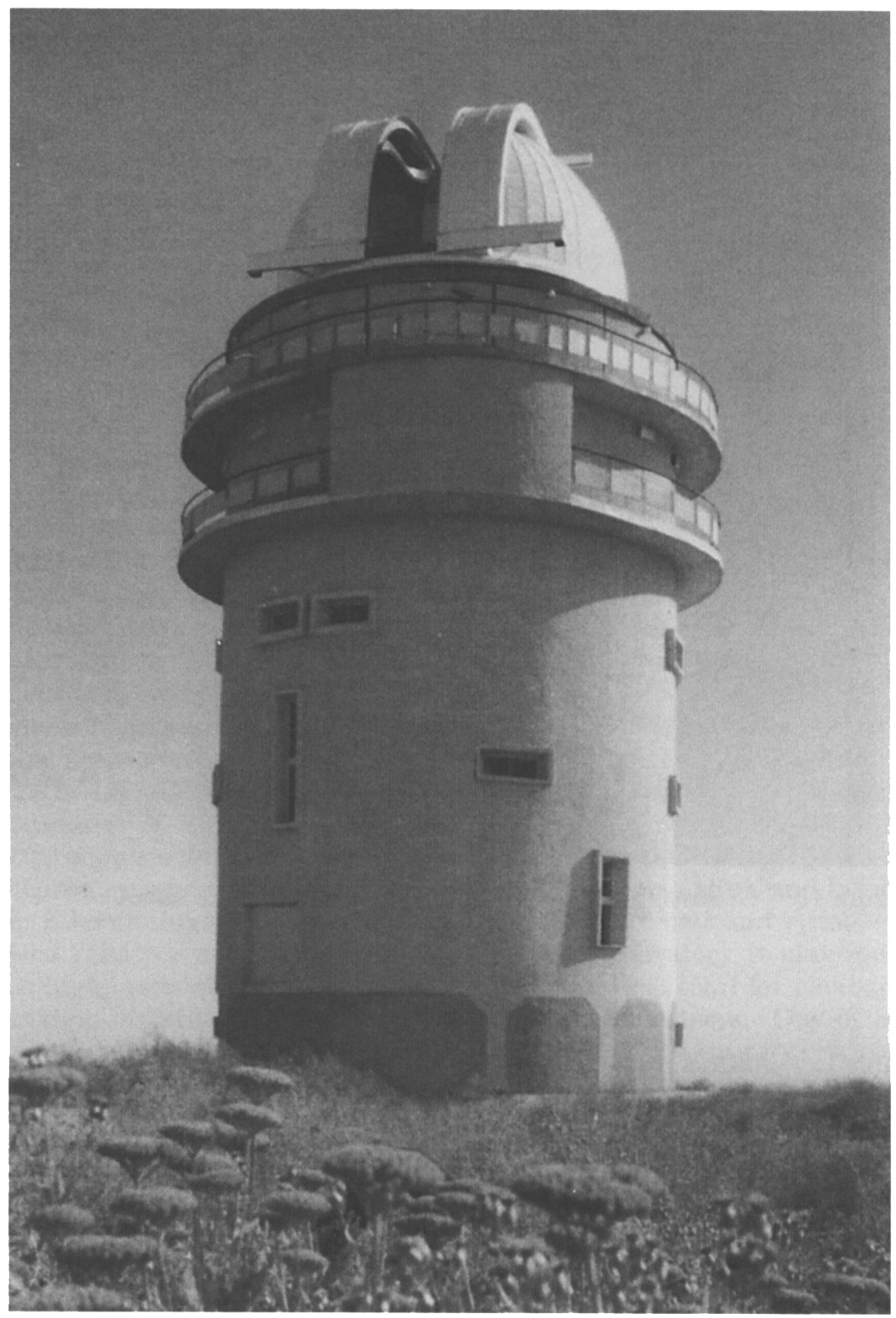

Figure 2. Sanglokh Observatory: Dome of the 1-m telescope 
Gorno-Badakhshan Autonomous Region of Tajikistan. There a 70-cm telescope RT 700 (with Cassegrain optical system) and a solar telescope are installed. Pamir observatory, with its unique astroclimate, is an excellent long-term site for astronomical submillimeter, IR and optical observations. There the average annual precipitation is less than $100 \mathrm{~mm}$ per year; monthly average wind-speed is about $6 \mathrm{~m} \mathrm{sec}^{-1}$; the brightness of the night sky in the V-band is less than 22 $\mathrm{mg} \mathrm{sec}{ }^{-2}$ (Kanaev et al., 1983). The basic astroclimatic characteristics of the astronomical observatories of Tajikistan are given in Table 1 in comparison to the data for some other observatories (Shevchenko, 1972).

Below we list some important scientific results obtained in the Institute of Astrophysics, Tajik Academy of Sciences, lately.

\section{Investigation of Meteors and the Earth's High Atmosphere}

The nature of meteoroids and the phenomena accompanying their flight in the Earth's atmosphere, the atmospheric trajectories, meteor radiation and ionization, the heliocentric orbits of meteoroids, distribution of meteor matter in the near-Earth space and, finally, the origin and evolution of meteoroid streams and meteor showers, are problems in the area of interest to scientists of the Institute of Astrophysics of the Tajik Academy of sciences.

Long-term complex (photographic, spectrographic, radar and TV) observations of meteors give extensive information about radiants and velocities, masses and densities, and orbits of many thousands of meteors. On the base of these observations the physical parameters and wind mode in the meteor zone of the Earth's atmosphere (heights $60 \mathrm{~km}-120 \mathrm{~km}$ ) have been determined; the contribution of meteors to the ionization of the high atmosphere and the influx of meteoric matter on to the Earth, Venus and Mars are studied; new meteor associations (i.e. groups of meteors with close orbital elements and radiants) have been revealed (Babadzhanov et al. 1990). From the results of photographic observations of meteors made from Dushanbe, catalogues of their orbits were compiled, which have been included in the data base of the IAU Meteor Data Center in Lund (Sweden).

In 1964-1965, in Dushanbe, a method of "instantaneous exposure" was devised. This method allows us, for the first time in the world, to obtain meteor photographs with exposures of $0.00056 \mathrm{~s}$. and, so, to establish the fact of fragmentation of meteoroids in the atmosphere. Later this method was used to obtain "instantaneous" meteor spectra as well (Babadzhanov, 1994).

Simultaneous photographic and radar observations of meteors, which have been carried out at the Gissar observatory, allow the specification of the correlation between intensity of meteor radiation and electron line-density of its train. According to radar observations of hundreds of thousands of meteors the spatial distribution of air flow in the meteor zone of the atmosphere and the processes of deionization of meteor trains were investigated.

During two years, (1968-1970), the Soviet Equatorial Meteor Expedition organized by the Institute of Astrophysics of the Tajik Academy of Sciences and the Kharkov Politechnic Institute worked in Somalia (East Africa) and, as results, the radiants and orbits of meteors of both the northern and southern hemispheres and the wind modes of the high atmosphere of the Earth's equato- 
rial area were determined. Processing of the observations obtained has provided fundamental astronomical and geophysical results, namely, a value for the influx of meteoric matter onto the Earth, the regularity of global circulation of the Earth's atmosphere at the heights of $80-120 \mathrm{~km}$, and a catalogue of orbits of several thousand faint meteors (Babadzhanov et al. 1974).

In the activity of scientists of the Institute of Astrophysics during the last 12 years, an important place was taken up by the large-scale investigation of the evolution of meteoroid streams and short-periodic near-Earth objects (comets and asteroids). As a result, a completely new conception of the evolution of both the shape of the meteoroid streams and the dynamics of meteor showers has been obtained. It was shown that the number of meteor showers produced by a comet depends on the Earth-crossing class of the cometary orbit, and that the majority of short-periodic comets are quadruple crossers of the Earth's orbit and, hence, each of them might produce four meteor showers. So, it was shown, that according to "meteor signs" a number of near-Earth asteroids, representing a danger to the Earth, are extinct cometary nuclei (Babadzhanov, 1998). Basic principles of meteoroid-stream formation and evolution developed in Dushanbe have served as the basis for a large series of scientific works carried out in other countries in recent years.

\section{Cometary Investigations}

The physics of comets is another important direction of scientific research at the Institute of Astrophysics, Tajik Academy of Sciences. These researches cover all sections of cometary physics and extensive observational and experimental material on comets has been obtained there. In 1955, A.M. Bakharev discovered a comet which became known as the Bakharev-McFarlane-Crinke comet. The expansion of the observational base and the use of telescopes installed in Gissar and Sanglokh observatories allowed spectroscopic, photoelectric, and polarimetric observations of comets to be carried out. In the 1950s a mechanical theory of cometary forms was developed, and problems of the interaction of corpuscular solar streams with cometary atmospheres have been investigated. A theory of plasma cometary tails was developed and non-stationary processes in comets have been also studied. It was shown that many such processes, for example cometary outbursts, are associated with solar activity (Dobrovolsky, 1966).

Our scientists have also developed a theory of the disintegration of cometary nuclei. The disintegration of cometary nuclei modeled with $\mathrm{H}_{2} \mathrm{O}, \mathrm{CO}, \mathrm{NH}$ and $\mathrm{CH}$ ices, and the snow model of a cometary nucleus, have been studied at the institute. The heat regimes of such nuclei were estimated. Theoretical data were tested under laboratory conditions which simulated the cosmic ones. The process of disintegration of dust matrices, the velocities of ejection of matrix fragments, and so on were studied in the laboratory. It was established that the nuclei of some short-periodic comets develop a refractory crust and they might evolve into asteroid-like bodies (Ibadinov, 1993).

Polarimetric and colorimetric investigations of comets have revealed negative polarization of the continuum radiation of comets at small phase angles (angle Sun-comet-Earth). Non-linear growth of cometary brightness in the region of small phase-angles has been found ("oppositional effect"). The bases of 
the theory of shock waves arising from the interaction of magnetized corpuscular solar wind with cometary plasma was worked out. The interaction of interplanetary dust particles with dust particles of the cometary atmosphere are being investigated and, a mechanism of X-ray radiation of comets and the occurrence of multicharged ions is proposed (Ibadov, 1996).

The Institute of Astrophysics of the Tajik Academy of Sciences actively collaborated in working up and in the realization of the International Halley Watch programs. Astrophysical and positional observations of a number of asteroids have been carried out and the orbital evolution of some Near-Earth asteroids associated with meteoroid streams, including the Taurid Complex asteroids, are being investigated.

\section{Astrometric Tasks}

In connection with the precise determination of the coordinates of the celestial objects and phenomena (meteors and comets, stars and asteroids, etc) astrometric methods were widely used and developed in the Institute. Furthere development of astrometic work in Dushanbe was connected with the launching of the first artificial Earth satellite (AES) (1957) and observation of such objects. A high-precision astronomical camera of the Gissar Observatory allows the making of photographic observations over a wide range of visible speeds.

\section{Investigation of Non-Stationary Stars and Galaxies}

Observations of variable stars in Tajikistan were made from the first days of the astronomical observatory in Dushanbe. Over the past years a "Sky Survey" has been created, unique photo-archives consisting of almost 70,000 sky negatives. From these some novae and more than 100 variable stars have been discovered in T-associations. In addition, some features of light-curves, the variability of periods of variables of different types, and oscillations in brightness of novae were studied. With the coming into operation of the Sanglokh 1-m telescope, photometric, polarimetric, and spectrographic observations of faint objects, extremely young stars, such as T Tauri and Ae/Be Herbig types, began to be made. Carrying out simultaneous polarimetric and photometric observations in different colors on some stars of the T-Tauri type, scientists of the Institute study physical conditions in the gas-dust shells of these stars (Borisov \& Minikulov 1997, Borisov \& Redkina, 1997; Redkina \& Zausaeva, 1997; Kiselev et al., 1995).

Theoretical investigations of the dynamical phenomena of the collective gravitational interactions of stars in galaxies have been widely developed at the Institute. Investigation of star formation in galaxies has shown that spiral waves of density in galaxies were active in producing not only the features of motion of stars and interstellar gas, but also the peculiarities of late star-formation processes. 


\section{Publications}

Since 1952, the Institute has issued the Bulletins of the Institute of Astrophysics of the Tajik Academy of Sciences. Up to the present time, 83 numbers have been issued. From 1958 till 1990 the Institute also issued the Soviet journal Comets and Meteors, up to its 40th number. Besides 12 monographs and thousands of scientific papers by the Institute scientists have been published in various places.

\section{Personnel and Training of Specialists}

The disintegration of the U.S.S.R., the social-political instability during 19911997 and the economic crisis in Tajikistan negatively affected the functioning of the Institute of Astrophysics: some buildings and structures of its Observatory were damaged seriously; in particular, the structures of Sanglokh Observatory. Now, none of the telescopes are supplied either with new radiation detectors (e.g., CCDs), or with old detectors (photographic plates and films). The scientific and technical personnel of the Institute have also suffered serious changes. Many highly qualified research workers have left Tajikistan, or transferred to more well-paid positions. Now, out the Institute's staff of 70, 20 are scientists, including four with the Dr.Sci degree and seven with the Ph.D. degree. Therefore, the Institute undertakes measures to restore its personnel.

Until recently there were two types of curricula for training astronomers: (1) in 1972-1988, an "astrophysics" specialization within the "Physics" curriculum in the Tajik State University; (2) beginning 1986, a "Physics and Astronomy" curriculum for schoolteachers in the Dushanbe Teachers' Training University.

On the initiative of the Institute of Astrophysics in 1999, a specialty of "Astronomy" and a Department of Astronomy were organized in the Tajik State National University. Twenty first-year students have finished the 1999-2000 academic year. The Institute of Astrophysics (and its observatories) has become the base research establishment for this specialization and expects an influx of capable youth into astronomy.

It is proposed to use the multistep system (Bachelor, Master, postgraduate studies) for training astronomers in Tajikistan. The structure of the astronomy bachelor curriculum is shown in Table 2 .

Table 2. Astronomy Bachelor Curriculum

\begin{tabular}{lc}
\hline Series of Subjects & Planned hours \\
\hline General humanitarian, economic and social & \\
disciplines & 984 \\
Mathematical and natural sciences disciplines & 1416 \\
General vocational and special disciplines & 2797 \\
\hline Total & 5197 \\
\hline
\end{tabular}

General vocational and special disciplines consist of the following courses: General astronomy

General astrophysics

Practical astrophysics 
Theoretical astrophysics

General astrometry

Celestial mechanics

Physics of the Sun and planets

Small bodies of the Solar system

Physics of stars

Physics of the atmosphere

History of astronomy

Galactic and extragalctic astronomy

Cosmology

Radioastronomy

Special laboratories and Practical astronomy

Now, for the training of personnel we need astronomical educational materials, such as sky atlases and maps, astronomical softwares, computers, etc.

\section{Prospects}

The prospect for astronomical research in Tajikistan depends mainly on the existing scientific and technical base, on training highly skilled scientific and engineering staff, on qualitative improvement of the scientific and technical base and on normal financing of the Institute.

In the next five years in the Institute and its observatories complex research on physics and dynamics of the Near-Earth Object (asteroids, comets, meteoroid streams and meteor showers), investigation of non-stationary stars etc. is planned for the Institute. The program of scientific research at Sanglokh Observatory, after its repair, envisages the following tasks:

- detection and investigation of extremely faint variables, members of Tassociations in the Galaxy and Cepheid variables in M31;

- complex photometric, polarimetric and spectroscopic investigation of the gas-dust shell of non-stationary stars of the T-Tauri type;

- observations of comets, near-Earth asteroids, meteor showers and fireballs.

The Institute has experience of work on international scientific programs and projects. In particular, important results were obtained there for the programs of the International Geophysical Year (IGY), the International Year of Quiet Sun, the IHW, etc. Together with Russian and Slovak astronomers a catalogue of orbital evolution of short-period comets was created. The Institute of Astrophysics of the Tajik Academy of Sciences and its observatories are open for scientific collaboration with scientists of other countries.

\section{References}

Babadzhanov, P.B. 1994, Asteroids, Comets, Meteors 1993, 45-54.

Babadzhanov, P.B. 1998, Cel.Mech. \& Dynam.Astronomy, 69, p. 221. 
Babadzhanov, P.B., Bibarsov, R.Sh., \& Kolmakov, V.M. 1990, Mass Distribution and the Flux of Sporadic Meteoroids, Moscow, $56 \mathrm{pp}$.

Babadzhanov, P.B., Kashcheyev, B.L., Nechitaylenko, V.A., \& Fedynskiy, V.V. 1974, Radar-meteor investigation of the high-atmosphere circulation, Publishing house "Donish", Dushanbe, 172 pp.

Borisov, Yu.V. \& Minikulov, N.K. 1997, Bull. Inst. Astrophys., Tajik Acad.Sci., No 83, p.19.

Borisov, Yu.B. \& Redkina N.P. 1997, Bull. Inst. Astrophys., Tajik Academy of Sci., No 83, p. 3.

Dobrovolsky, O.V. 1966, Comets, Moscow, Nauka, 288 pp.

Ibadinov, Kh.I. 1993, Doklady Academii nauk Resp. Tajikistan, XXXVI, No 3, p.182.

Ibadov, S. 1996, Physical Processes in Comets and Related Objects, Moscow, Kosmoinform Publ. Comp., 181 pp.

Kanaev, I.I., Sholomitsky, G.B., Maslov, I.A., \& Grozdilov, V.M. 1983, Review of Science and Technics (Series of "Astronomy"), Vol. 22, p. 286.

Kiselev, N.N., Minikulov, N.K., \& Chernova, G.P. 1995, Bull. Inst. Astrophys., Tajik Acad. Sci., No 82, p. 42.

Redkina, N.P. \& Zausaeva O.G. 1997, Bull. Inst. Astrophys, Tajik Acad. Sci., No 83, p. 7.

Shevchenko, V.S. 1972, Young Star Complexes. Astroclimat, Publ. house "Fan", Uzbekistan, p. 84.

\section{Discussion}

In answer to a question, Babadzhanov stated that salaries of astronomers in Tajikistan are not in arrears. 